\title{
Organization and management of the International Space Station (ISS) multilateral medical operations
}

\author{
J. M. Duncan \\ National Aeronautics and Space Administration Lyndon B. Johnson Space Center; \\ 2101 NASA Road 1, Houston, TX 77058, USA \\ V. V. Bogomolov \\ Institute for Biomedical Problems, Russian Academy of Sciences, State Research Center of the Russian Federation; \\ 76-A Khoroshevskoye sh.,Moscow, 123007, Russia \\ F. Castrucci \\ European Astronaut Centre, European Space Agency; Linder Hoehe, 51147 Cologne, Germany \\ Y. Koike \\ Japan Aerospace Exploration Agency, Tsukuba Space Center; 2-1-1 Sengen, Tsukuba-shi, Ibaraki 305-8505 Japan
}

J.M. Comtois

Canadian Space Agency; 6767 route de l'Aeroport, Saint-Hubert, Quebec J3Y $8 Y 9$ Canada

A.E. Sargsyan*

Wyle Laboratories Life Sciences Group; 1290 Hercules Dr. Ste 103, Houston, TX, 77058 USA

\begin{abstract}
The goal of this work is to review the principles, design, and function of the ISS multilateral medical authority and the medical support system of the ISS Program. Multilateral boards and panels provide operational framework, direct, and supervise the ISS joint medical operational activities. The Integrated Medical Group (IMG) provides front-line medical support of the crews. Results of ongoing activities are reviewed weekly by physician managers. A broader status review is conducted monthly to project the state of crew health and medical support for the following month. All boards, panels, and groups function effectively and without interruptions. Consensus prevails as the primary nature of decisions made by all ISS medical groups, including the ISS medical certification board. The sustained efforts of all partners have resulted in favorable medical outcomes of the initial fourteen long-duration expeditions. The medical support system appears to be mature and ready for further expansion of the roles of all Partners, and for the anticipated increase in the size of ISS crews.
\end{abstract}

\section{Keywords}

International Space Station (ISS), space medicine, human space flight, medical certification, international cooperation

\section{Introduction}

The current stage of human spaceflight is characterized by unprecedented technological advancements and broad international cooperation in the assembly and operation of the International

\footnotetext{
${ }^{*}$ Corresponding author.

E-mail address: asargsyan@wylehou.com (A.E.Sargsyan); (1-281) 212-1263 (fax)
} 
Space Station (ISS). Medical support is among the areas where synergistic inputs from all Partners (Russian Federal Space Agency (FSA), the U.S. National Aeronautics and Space Administration (NASA), the European Space Agency (ESA), the Canadian Space Agency (CSA), and the Japan Aerospace Exploration Agency (JAXA)) have resulted in a robust and highly effective multilateral system.

Up to the mid-1990s, the medical support systems for human space flight in Russia and the U.S. differed substantially. However, the founding documents of the ISS Program called for integration of medical support through integrated crews, a common system of medical support, and coordination of crew health matters on the principle of consensus.[1,2] Efforts and resources of all Partners were to be coordinated for the development and operation of a joint system that would best maintain health and performance of international crews in all phases of ISS missions. In order to overcome the differences and integrate the national systems of medical support, a number of objectives were identified, first during the phase of preparation and implementation of the U.S.Russian Shuttle-Mir and NASA-Mir projects [3,4,5], and later within the framework of multilateral cooperation for ISS. These objectives included reduction and mitigation of risks associated with space flight factors that were distinct in their nature, as well as establishment of environmental conditions in habitable compartments of the spacecraft that were conducive to healthy and productive life and work. It was particularly important to provide readily available, effective means and methods of inflight countermeasures to mitigate the well-known adverse effects of long-duration space flight on crew health [6-8]. Water, food products, air, and the station interior had to be verifiably clean, healthy, and free of toxic impurities and microbiological contamination. Risks had to be predicted and analyzed for nominal flight conditions (launch, dynamic on-orbit operations, EVA, implementation of complex flight programs, and crew return), as well as for the occurrence of various off-nominal situations such as malfunctions of station engineering systems including life support, and failures of medical and exercise equipment. An acceptable crew work/rest schedule had to be worked out, balancing work, exercise, personal time, and sleep, since crew fatigue is known to be a leading cause of performance decrements and operator errors [9-10]. Finally, the station was to be equipped to identify and treat predictable medical situations on orbit, and to provide means for safe medical evacuation in case of life-threatening emergencies.

Thus, notwithstanding the experience accumulated in all pre-ISS programs and broadly available through many review publications, creation of a common, consensus-based system for medical support of joint crews posed a substantial challenge. Utmost regard and respectful consideration was given by each partner for the knowledge and experience of the others, as well as to their established regulations and procedures for crew medical support. Many large and small 
obstacles were encountered and overcome to achieve integration and standardization. The cumulative effect of the many previous years of international cooperation in space biology and medicine, along with the leadership of the space medicine managers and experts of all Partners, created favorable conditions for continued cooperation and integration. A number of developments were highly contributory to the development of an effective ISS crew medical support system: interaction and joint operations by NASA and FSA medical communities in the Phase 1 program[4,5]; the establishment and operation of the ISS medical boards, panels and working groups; the development of the common ISS medical requirements; adoption of common ISS medical selection standards and evaluation requirements; the joint certification of the first four ISS expedition crews; exchanges of personnel including flight surgeons, and the adoption of the concepts of the Integrated Medical System (IMedS) onboard and the Integrated Medical Group (IMG) on the ground. The joint efforts of all the Partner medical organizations resulted in an effective international system of operational medical support.

\section{Main documents regulating international cooperation on the ISS}

The cooperation in construction and operation of the ISS was initiated and authorized by the Agreement Among the Government of Canada, Governments of the Member States of the European Space Agency, the Government of Japan, the Government of the Russian Federation, and the Government of the United States of America Concerning Cooperation on the Civil International Space Station, signed in January of 1998 (the Intergovernmental Agreement, or IGA)[1]. The IGA recalls all international space treaties that led to the ultimate development of an international partnership to build and operate the ISS. It recognizes the Russian Federation's "unique experience and accomplishments in the area of human space flight and long-duration missions, including the successful long-term operation of the Russian Mir Space Station." The IGA further stipulates that the United States has the leading role for overall ISS management and coordination, and that detailed provisions for implementation of the agreement would be developed in Memoranda of Understanding (MOU). These MOUs were subsequently developed and signed as bilateral agreements between NASA and each ISS partner, i.e. the Russian Space Agency, the European Space Agency, the Canadian Space Agency, and the Government of Japan.

The basic principles governing the ISS crew health matters are defined in Article 11.4 of each ISS MOUs [2] (Appendix). This Article authorizes three multilateral forums of medical authority, namely the Multilateral Medical Policy Board (MMPB), the Multilateral Space Medicine Board (MSMB), and the Multilateral Medical Operations Panel (MMOP), and provides a framework for 
consensus-building based on discussion and negotiation, which proved to be viable over time.

Conflict resolution mechanisms built into the IGA and MOU's to assure decision-making capacity of the multilateral groups. However, decisions of medical nature, such as those on space flight medical eligibility of particular individuals, were to remain the prerogative of medical specialists.

\section{ISS Multilateral Medical Authority Structure and Medical Policy Framework}

As noted above, the Article 11.4 of the ISS MOUs establishes a multilateral medical authority structure and calls for a joint system of medical support. The entire ISS medical organization is designed on the premise of full cooperation and active participation of all partner members. The MMPB, the MSMB, and the MMOP are the original groups expected to further develop and implement policies, standards, requirements, and operational mechanisms in the framework of the ISS partnership. Groups and forums were thus established to create a system of operational medical services for the ISS. Charters of the three multilateral groups became the initial vehicle to implement the guidance of the MOUs. A considerable effort was applied by all Partners to define roles, responsibilities, and primary functional features of these groups. Considerations were addressed regarding the sensitive nature of the medical authority and the need for decision-making capacity based primarily on medical and safety considerations, without undue bias or influence. The ISS medical authority structure is depicted in Figure 1.

\subsection{The Multilateral Medical Policy Board (MMPB) and ISS Medical Policy}

The MMPB is the highest level of medical authority responsible for medical policy formulation and oversight of all crew health-related matters. While members of the MMPB are answerable to their respective agencies, the Board as a whole exercises its authority independently of the ISS management bodies and operates on the basis of coordinated decisions (consensus). The NASA and FSA members of the MMPB co-chair the Board until the ISS assembly is completed. MMPB issues medical policy decisions through special Policy Directives, statements in meeting Minutes, letters, and review of high-level ISS documents, such as charters, sets of standards, and requirements. A most prominent feature of MMPB has been its adherence to the fundamental principles discussed below.

\subsubsection{Integrated approach to medical policy: With a goal to ensure the best possible health} outcomes for the ISS crew, the MMPB explicitly pronounced an integrated approach to medical policy, requiring and upholding an integrated health support system operating on the best available evidence, with the best available resources and highest ethical standards, as a component "essential to the success of the ISS". 


\subsubsection{Evidence-based, scientific approach to the operation of the ISS medical support}

system: The MMPB has made a commitment to supporting International Partners' cooperative work "to reach a common understanding based on the best available scientific evidence... with the goal of making informed decisions." Furthermore, the favorable health outcomes of human space flight in both the U.S. and the Russian space programs was seen as evidence of the success of both systems, which formed a basis for continued progress in the development and operation of the new medical support system. Scientific evidence from both space and terrestrial medical practice and research is broadly used throughout the ISS medical community, from medical certification of crewmembers to in-flight countermeasures and environmental health assurance, to postflight rehabilitation of longduration crews.

\subsubsection{Common Space Medicine Standards. Early in the ISS operations, the ISS medical} policy supported adherence to the existing space medicine standards already agreed upon. MMOP and MSMB undertook the development of common space medicine standards in a broader interpretation, including not only medical certification standards, but also requirements for training and certification of flight surgeons and other medical support personnel, diagnostic protocols, countermeasures, treatment protocols, environmental standards, post flight rehabilitation, and radiation standards for the station. In the areas without common standards, however, the standards of each Partner have been recognized until common standards are developed.

3.1.4 Ongoing Integrated Medical Support. The MMPB worked to establish principles of medical support and medical responsibility in nominal ongoing operations, in a degraded support capability, and in medical contingency situations. In nominal operations, the ISS Mission Control Center - Houston (MCC-H) is the controlling center, with the Crew Surgeon responsible for the medical care of all individuals on the ISS in cooperation with the crew surgeons assigned to a given ISS Increment by Partners having crewmembers onboard. When ISS control is passed to another mission control center, medical responsibility is also assumed by that center. For example, the Mission Control Center - Moscow (MCC-M) took control of ISS support during a 2005 closure of the MCC-H due to a tropical hurricane. Non-emergent ISS contingencies of medical relevance, including illness or injury, are addressed by the Integrated Medical Group (IMG), an international medical group distributed among the active MCCs. Operational medical management supervises and directs the IMG through Space Medicine Operations Team (SMOT), a weekly private medical forum by treleconference.

In operational situations where the capability of one or more ISS health support systems is degraded, the combined resources of all Partners' components are considered in determining the ability of the remaining ISS systems to support the health and safety of the ISS crew. To facilitate 
this process, a commitment has been made and followed to share all necessary information among Partners in a timely fashion. Furthermore, consistent with the humanitarian principles of medical care, the ISS Program has expressed determination to make any on-orbit medical capability available to all crewmembers as appropriate, and to cooperate in the analysis of samples and data. Nonemergent medical situations with potential major operational impacts, such as situations requiring medical evacuation, are to be reported to the highest-level medical authority (MMPB) for review. However, in the event of a medical or health-threatening emergency onboard the ISS, in which there is insufficient time for consultations, the Crew Surgeon at the controlling MCC has the authority to choose the medical response and directly communicate the appropriate recommendations to the ISS Increment Flight Director.

\subsubsection{Consensus and Issue Resolution. The ISS medical policy requires every effort to} achieve consensus in medical and health decisions to best support the health of the crew. With the principle of consensus in foremost consideration, and in keeping with NASA's responsibility for overall Space Station management and safety, a detailed process for dispute resolution was adopted, but has never been invoked to the present time. Generally, all Partner medical organizations are determined to achieving final and satisfactory conflict resolution and avoiding referral of medical decisions to non-medical management authorities.

\section{ISS Medical Requirements and Standards}

The development and implementation of written medical requirements based on the ISS IGA and MOUs is a complex, multi-dimensional process. During this process consideration is given to space and terrestrial medical knowledge and evidence, medical risk estimates, accepted standards of care in the Partner countries, engineering and planning considerations, as well as crew expectations and concerns. In light of these complexities, there has been an ongoing dialog between the medical community and the Program management to define the medical requirements for long duration space flight as accurately and completely as possible. The baseline version of ISS Medical Operations Requirements Document ("ISS MORD”) was developed during the pre-operational period of the ISS medical organization (1997-1998), and was approved by the MMOP and the ISS Program in September of 1998. The first updated revision (Rev. A) was approved by all Partners and published by the ISS Program soon after the beginning of piloted ISS flight (December 2000). Four interim updates were published to this version between July 2002 and May of 2003. In August of 2006, a new revision of medical requirements was adopted by the ISS program for implementation. The ISS medical organization redefined the requirements in a most unambiguous language complete with a 
rationale and definition of "consequences of non-implementation". Sustained efforts are still necessary to continuously upgrade the ground and ISS infrastructure of medical support to a more robust and sufficiently redundant level, in order to support a crew of six individuals without reductions in the ISS medical risk mitigation capacity.

Substantial discordances among all ISS Partners' approaches to a variety of health and medical issues, including medical selection standards and certification criteria, prevented rapid development of joint medical standards. Following negotiations, in June of 1998 the MMOP adopted the NASA Astronaut Medical Evaluation Requirements Document (classified as AMERD 2A) as a temporary common standard for all Partners; a respective official protocol was signed. Few exceptions were defined in agency-specific Matrices, most of which referred to additional requirements levied by each Partner on their crewmembers. Several evaluation selection tests and examination were substituted by alternative methods. This management decision was necessary to enable the multilateral medical certification process by the MSMB. However, it soon became apparent that an updated set of common standards and evaluation lists was necessary to take advantage of the entire space medicine experience and the advances in modern evidence-based medicine. Furthermore, the need arose to define specific criteria and requirements for the selection of Space Flight participants (tourists), as the Russian space agency began offering seats on Soyuz vehicles to individuals that were not professional cosmonauts or astronauts.

The first document of the new series received MMOP approval in May of 2002, and was entitled "Medical Standards and Certification Procedures for Space Flight Participants". These standards were designed for individuals transported to the ISS for commercial visitation or other short-term purposes (less than 30 days), generally fare-paying passengers without operational duties or assignments. The new multilateral version of the standards for long-duration ISS crewmembers was approved in 2005-2006 as "Medical Evaluation Document Vol. A and B". The document includes causes for rejection, health and anthropometric standards, as well as medical evaluation requirements for selection and all phases of ISS missions.

Flight Rules comprise a part of the ISS requirements documentation. The Flight Rules outline decisions planned in advance to minimize the amount of real-time discussion. Their importance is especially evident in view of the multilateral nature of the ISS Program. The ISS Flight rules are thoroughly coordinated documents organized in four volumes: Volume B (ISS Generic Operational Flight Rules), Volume C - Joint Shuttle-ISS Operational Flight Rules, and Volume D (Soyuz/Progess Joint ISS Flight Rules). The majority of medically relevant flight rules, grouped in the "Aeromedical" sections (B-13, C-13 and D-13), are related to foreseeable limit violations (such as oxygen concentration in the respirable air), constraints or anomalies, and to mitigation of related medical 
risks. Some flight rules are concise and self-explanatory, while others may be divided into sections and may contain an elaborate rationale and a list of references. All rules are maintained in both English and Russian languages, to ensure their immediate availability for real-time operational use.

\section{The Multilateral Space Medicine Board (MSMB) and Crew Medical Certification}

MSMB is a medical group comprised of 5 primary members (one from each agency). Alternate members are also identified. The role of this board is primarily clinical, and includes medical certification of the ISS crew and visiting crewmembers for space flight to ISS. MSMB is also responsible for qualifying ISS flight surgeons from all Partners.

The MSMB determines the medical eligibility of ISS crewmember candidates for training (including hazardous training activities) and long-duration missions, in accordance with medical standards adopted in the MED Vol. A document. The MSMB also medically certifies all persons visiting the ISS aboard the Soyuz and Shuttle missions for the duration of docked operations, including Space Flight Participants (individuals other than professional astronauts or cosmonauts, also referred to as "tourists"). Each partner selects and certifies their crewmembers via their Agency's Medical Board, and submits the individual medical data for approval by the MSMB. In its certification process, the MSMB follows the overarching principles of full disclosure of medical data, thorough clinical review with extensive use of available terrestrial and space medical evidence, and decision-making by consensus.

Concerns that arise are resolved via clinical discussion (meetings, teleconferences, and electronic communications), inviting the assistance of clinical experts from various countries when necessary. In all such occasions, medical decisions are made within the medical groups. It is not uncommon for the MSMB to hear evidence review presentations in order to assess the probability of disease manifestations and the magnitude of medical and mission consequences in a given case, and to determine whether the combination of the risk and its available mitigation warrants justifiable clearance for the mission. By May of 2007, the MSMB had held ?? meetings and teleconferences, to document ?? medical certification events (?? certification decisions for new candidates, ?? recertifications, and ?? temporary extensions of medical certification status), resulting in an average number of certification events of ?? per individual.

\section{The Multilateral Medical Operations Panel (MMOP) and ISS Medical Operations}

MMOP is the operationally oriented panel with one member from each agency. The MMOP is responsible for development of medical requirements, clinical standards, implementation plans, and operational procedures. The MMOP has dual reporting responsibilities: to the MMPB for issues 
related to medical policy and to the ISS Program Office (Mission Integration and Operations Control Board, MIOCB) for operational issues. The MMOP assembles monthly by a videoconference and operates on the principle of consensus. It is assisted by 11 multilateral Working Groups of special expertise (see Fig. 1: ISS multilateral medical authority structure.) Some of the important MMOP tasks are: control of the ISS Medical Operations Requirement Document (MORD), development of ISS medical standards, development of training requirements and guidelines for ISS crewmembers and flight surgeons, continuing evaluation of medical and environmental monitoring capability, and approval of mission medical readiness of Shuttle or Soyuz launches to the ISS. The MMOP Working Groups include representation from all agencies with competence in the corresponding fields. The Working Groups perform a detailed analysis and discussion of issues and provide recommendations to the MMOP, thus playing a key role in introducing special partner approaches to the multilateral medical organization.

\subsection{The Approach to ISS Medical Status Review and Prognostic Evaluation}

The system of continuous mission status review and prognostic evaluation is an important feature of the ISS medical support. The ISS Program's medical organization has created an process of health-related information review that routinely combines recent data and anticipatory considerations on a daily, weekly, monthly, and per-increment basis (Fig. 3). The system maintains a firm grip on all the factors that allow timely identification and mitigation of medical and health risks. For every given moment, the medical status of the station and its crew has been previously addressed in a "forward-looking" fashion by the previous expedition readiness review, a previous monthly "look-ahead" review, the preceding week's SMOT teleconference, and the daily interactions within the Integrated Medical Group (IMG). This continuous proactive process hinges on multilateral participation.

\subsubsection{Daily activities and status review (IMG)}

Depending on the mission schedule, various medical operations activities may be scheduled for a given day, such as medical and psychological conferences with the crew, medical testing, or IMedS data downloads and analysis. At the daily level, no formal multilateral events are conducted, and most activities involve only partners having a crewmember onboard. The Crew Surgeon and Partner flight surgeons at the active MCCs are fully aware of all the activities and work as a virtual single group called the Integrated Medical Group (IMG). Environmental, radiation and similar data are shared as soon as they become available. Preparations are made for the events anticipated in the following days 
or weeks, such as pre-EVA planning, exercise regimen adjustments, or sleep shifting coordination. The daily data are thoroughly documented and a daily status report is completed by the missionassigned biomedical engineers. The medical operations plans are coordinated with the ISS mission planning community and the engineering community if necessary. The Crew Surgeon or Deputy Crew Surgeon is available at all times for immediate communication with mission management, the crew, any mission support personnel, or the medical management of any Partner. The ISS medical organization thus maintains, through the IMG, a current knowledge of the status of the crew health and activities, and is prepared for the activities of the following days.

\subsubsection{Weekly status review: Space Medicine Operations Team (SMOT)}

Since the beginning of piloted operations, Partners established a weekly operational medical forum by audio teleconference. Called SMOT, this forum is attended by the IMG flight surgeons and the operational medical management of all Partner agencies. Since the first event on November 08, 2000 , this forum has assembled weekly with no interruptions. With attendance limited to physicians and essential personnel, this is the only multilateral forum besides the MSMB certification events where medical issues are disclosed and openly addressed will representatives of all Partners.

Each SMOT meeting follows a formal agenda that includes recurring Items (status reports), new items or concerns (issues submitted for discussion by any Partner), and announcements. Status reports from MCC-H are presented by the Crew Surgeon (or Deputy Crew surgeon), and the Partner flight surgeons. Standard report from MCC-M is provided by the Lead of the Group of Medical Support, and primarily focuses on the review of the Russian components of medical operations and data obtained through the Russian orbital segment. It is important to note that this report integrates information, positions, and concerns from all the medical organizations of the Russian partner, thus representing a unified Russian input.

During the SMOT teleconferences that coincide with Soyuz or Shuttle flights to ISS, status reports are heard not from one, but two Crew Surgeons (arriving and departing crew), and surgeons assigned to the visiting crews or particular crewmembers. Such reports may be delivered from other locations, such as the NASA Kennedy Space Center or the Gagarin Cosmonaut Training Center in Russia. Such expansion of the SMOT is driven by the principle that during docked operations the ISS medical support system is primary, and the ISS Crew Surgeon is the lead of the IMG complement regardless of its current composition.

At the end of each SMOT, the Partners cast a formal vote for approval of nominal ISS operations in the following week, based on medical considerations. While the complete SMOT 
Protocol is documented and archived in a secure fashion, a "de-privatized" version, stripped of confidential data, is available to any working-level medical operations personnel to ensure the currency of their knowledge of the medically relevant circumstances of the mission.

The SMOT, by the virtue of its unrestricted access to any and all information related to crew health on the one hand, and the authority of the medical managers present, on the other, is seen as the main pathway for short-term operational input into the ISS mission management through the ISS Mission Management Team. Such inputs can be made through a delegate to the MMT meeting, by special written communication on behalf of SMOT, or through the official SMOT protocol (with private data omitted), reflecting the concerns and recommendations. By May of 2006, SMOT had conducted over 330 weekly teleconferences.

\subsubsection{Monthly status review: Anticipatory (look-ahead) Medical Operations Status Reviews}

Beginning with ISS Expedition 9, the partners established a monthly anticipatory (lookahead) routine procedure to address operational situations and conditions for the near-term medical support of the ISS crew. These mandatory monthly reviews are scheduled in conjunction with the SMOT teleconferences. Any foreseeable factors that may disrupt the nominal progress of the mission from the medical perspective, or compromise the quality or adequacy of operational medical support, are brought up and discussed. Generally, they follow an SMOT teleconference and consist of reports that identify special operational demands and circumstances of the following month, and estimate the degree of readiness of the IMedS and IMG to meet such demands. Examples have included

predictions regarding exercise equipment life cycle limits, ambient noise concerns, or needs of pharmacy replenishment that would claim increased up-mass allocation. This activity has proven to be highly beneficial, as it mobilizes the medical operational personnel to be proactive and consider a longer-term outlook of all the circumstances surrounding the medical support of ISS mission.

\subsubsection{Integrated MMOP Review of Mission Medical Readiness}

After completion of agency-level readiness reviews, the MMOP Mission Readiness Review event is conducted (generally matched with the last monthly "look-ahead" review of the previous expedition, or an MMOP meeting). Using a formal Launch Readiness Checklist, Partners develop a formal position of the medical organization regarding readiness for the next ISS increment (mission) in an integrated fashion. The resulting Launch Readiness Statement to the ISS Program addresses medical aspect of the upcoming mission, explicitly confirms availability of the required capabilities, and notes exemptions or exceptions. The Statement includes requests to the ISS Program regarding 
high-priority aspects of the mission, or special concerns from the medical perspective (Table 1). The Launch Readiness Statement is a culmination of the multi-level continuous anticipatory reviews system. The MMOP generates readiness statements for non-crew exchange missions as well, if they have any medical implications such as visiting crew flight to ISS).

\subsection{The Integrated Medical System (IMedS)}

Although medical and countermeasures hardware and inflight medical operations plans are originally divided by origin (supplying agency), they are utilized and executed in an integrated fashion. The policy-level support of the medical system integration allowed the entire medical support infrastructure aboard the Station to be treated as the ISS Integrated Medical System (IMedS). The realization of the IMedS concept united the NASA Crew Health Ccare System (CHeCS) as overseen by the Johnson Space Center, the Russian medical hardware as overseen by the Russian Medical Support Group, and conceivably other Partners' future medical equipment, under a single unified working concept. When evaluating medical aspects of new mission plans (including mission readiness review), medical personnel presents the results to the ISS Program in an integrated fashion, meaning decisions have already been coordinated among all ISS medical counterparts. Joint procedures are integrated into the common Station Operations Data File books, and ground documentation such as the ISS Medical Hardware Catalog, which consolidates all medical equipment descriptions into a single volume.

In similarity with the medical support hardware, the ISS medical kits with medication, medical and dental tools and examination devices, dressings, splints, and other small portable medical items, are the responsibility of the agency of origin but united in the same concept of integrated medical assets. The medical items of all origins are always considered when a medical capability for a given situation is assessed.

The capabilities of the IMedS are remarkably robust when compared with any previous space program. Diagnostic equipment, besides electrophysiology and pulmonary function testing, includes a large number of blood and urine tests. For a medical contingency requiring imaging support, the IMedS can be augmented by a multipurpose ultrasound system available in the ISS Human Research Facility. For the first time in the history of human space flight, diagnostic imaging has become a required capability for medical risk mitigation $[11,12]$.

\subsection{Behavioral aspects of ISS missions}


Any deterioration in behavioral health and performance, especially at critical operational points in the mission, may have direct impact on the mission's safety and success $[13,14]$. Ineffective medical and behavioral support could increase the mission and medical risks due to fatigue, task overload, illness, low morale/motivation, or interpersonal frictions within the crew or between the crew and the ground personnel. Such deterioration may be insidious and may remain unnoticed for a long time, to present itself at a critical, unforgiving point when the demands for accuracy and efficiency are the highest.

Successful behavioral health and performance monitoring and intervention require specialized expertise that is not always part of a physician's training. Therefore, the crew-assigned flight surgeons are assisted by behavioral health and performance specialists, who have been regularly involved with the crewmembers through all phases of the mission. MMOP is supported by a Spaceflight Human Behavior and Performance Working Group (SHBPWG) since 1998, to coordinate Partner inputs regarding behavior, performance and psychological factors and requirements for longduration space flight, psychological characteristics and needs of multicultural crews, and crew psychological training and support. The vast experience and immediate operational involvement of its members in their home agencies allowed the group to not only respond to MMOP actions, but also to proactively generate recommendations to the MMOP in the area of behavioral support and crew protection.

The psychological state of the crewmembers and their performance has long been known to depend, among other factors, on their work-rest schedule, and maintenance of a stable circadian rhythm.[17] The ISS medical organization makes consistent efforts to set limits to daily working hours, and to protect the personal time of crewmembers. Crew work time demands tend to sharply increase during docked operations and the preceding several days, around the time of cargo spacecraft arrivals, in cases of off-nominal conditions and system failures, and EVA events. Same events usually require circadian shifting, further complicating the crewmembers' ability to rest and recuperate from intense work activities. Every such instance requires extensive coordination among the IMG personnel with subsequent integration of the medical decisions into the crew timeline.

\section{Conclusions}

The ISS assembly and operation in the piloted mode has been ongoing for over 6 years, partly delayed due to various setbacks. Currently, Expedition 15 is working aboard the station. The duration of expedition crew stays on orbit has ranged from 129 to 196 days. Throughout all of the ISS expeditions, the medical requirements were essentially met and medical operations were performed in accordance with multilaterally adopted documents, particularly the ISS MORD. The structure 
established to manage medical operations permitted adequate response to occurring problems, thereby assuring crew health and a level of performance required to perform mission tasks. No serious clinical problems among crewmembers were observed neither aboard the station, nor in broader terms of the mission medical outcomes. ISS life support systems were sufficient and adequate, and intermittent temporary failures of individual equipment did not affect crewmember health.

The environmental conditions on the ISS during all flights have, on the whole, remained satisfactory and in conformance with the regulatory requirements of the ISS MORD. Some concern was elicited by noise levels in certain areas of the station and by the occasional need to suppress microbial and fungal contamination of surfaces. The methodology developed to monitor the station's environment provided adequate objective information to ensure healthy living and working environment for the crew. At the same time, addition of new modules and arrival of multiple cargo and transport vehicles tends to increase the integral contamination of the station air.

The vast majority of the scheduled medical operational activities were completed. Failures of medical hardware were encountered, however without substantial impact on health, safety, or performance of the crews. The U.S. and Russian contributions to the IMedS, and the real-time mission operations have been partly redundant and largely complementary, and have ensured uninterrupted provision of essential medical services to the ISS Program. Expedition 14 marked the beginning of transition from bilateral to multilateral contribution, as the European Partner provided a long-duration crewmember ( $\mathrm{T}$. Reiter), and added it mission control capability to the previously bilateral mission control infrastructure. The The ISS Program apprears to be ready for further expansion of the roles of all ISS Partners, the eventual crew increase to six individuals, and to the assembly-complete utilization of the ISS. 


\section{Acknowledgements}

The authors highly regard the leadership, wisdom and relentless efforts of the international groups and individuals that outlined the policy framework of the ISS joint medical support and laid the foundation of the system that is operating today. Whether representing their agency in the design phase of the Space Station, or serving on the medical authority groups such as MMPB, MSMB, and MMOP, they have made great contributions to a truly historical undertaking - a sophisticated but highly functional international medical system of continuous operational nature.

\section{Appendix}

11.4 NASA, CSA and other Partners will establish a Multilateral Medical Policy Board (MMPB) to provide coordination and oversight of crew health issues. NASA and CSA will each provide single point of contact for medical support who will have full responsibility to resolve issues related to the development of a common system for medical support. The MMPB will be supported by a Multilateral Space Medicine Board (MSMB) and by a Multilateral Medical Operations Panel (MMOP), established by NASA and CSA with the other Partners, which will be the primary working level groups for coordination of crew health matters including clinical care, medical standards, preventative medicine (including operational countermeasures) and environmental monitoring. The MMOP and the MSMB will operate on the principle of consensus. The MMOP will develop medical standards, certification criteria, pre-flight, in-flight, and post-flight medical care requirements, medical hardware responsibilities and operational procedures and recommend them to the MSMB for approval. The MSMB will present its decisions and findings to the MMPB and MCOP, as appropriate, for review and concurrence. NASA, CSA, and the other Partners will be responsible for medical certification of their respective crew member in accordance with agreed standards, and will present the appropriate documentation to the MSMB for approval. The MSMB will have responsibility for final medical certification of crew and for oversight of the implementation of medical operations.

Authors' Note: Article 11.4 in all four ISS MOU's are identical, with the exception of the Partner's name. 


\section{References}

[1] Agreement Among the Government of Canada, Governments of the Member States of the European Space Agency, the Government of Japan, the Government of the Russian Federation, and the Government of the United States of America Concerning Cooperation on the Civil International Space Station. Washington., D.C., January 1998.

[2] Memorandum of Understanding Between the National Aeronautics and Space Administration of the United States of America and the Canadian Space Agency on the Cooperation on the Civil International Space Station. Washington., D.C., January 1998.

[3] Gontcharov IB, Kovachevich IV, Pool SL, Navinkov OL, Barratt MR, Bogomolov VV, House N. In-flight medical incidents in the NASA-Mir program. Aviat Space Environ Med. 2005 Jul;76(7):692-6.

[4] Goncharov I.B., Kovachevich I.V, Pool S.L., Novinkov O.L., Barratt M.R. Medical Care System for NASA - Mir space flights.// Aviation, Space, and Environmental medicine 2002 Dec;73(12):1219-23.

[5] Morgun VV, Voronin LI, Kaspranskiy RR, Pool SL, Barratt MR, Navinkov AL. Development of U.S.-Russian medical support procedures for long-duration spaceflight: the NASA-Mir experience. Aviat Space Environ Med. 2002 Feb;73(2):14755.

[6] Gazenko O.G., Grigoriev A.I., Kozlovskaya I.B. Mechanisms of acute and chronic effects of microgravity. Physiologist. 29: S48-S50.1986

[7] Kozlovskaya I.B. Countermeasures for long-term space flights. Lessons learned from the Russian space program, Journal of Gravitational Physiology 9 (1), 313. 2002

[8] Leblanc AD, Spector ER, Evans HJ, Sibonga JD. Skeletal responses to space flight and the bed rest analog: A review. J Musculoskelet Neuronal Interact. 2007 JanMar;7(1):33-47.

[9] Caldwell JA. The impact of fatigue in air medical and other types of operations: a review of fatigue facts and potential countermeasures. Air Med J. 2001 JanFeb;20(1):25-32. Review.

[10] Christensen JM, Talbot JM. A review of the psychological aspects of space flight. Aviat Space Environ Med. 1986 Mar;57(3):203-12. Review.

[11] Sargsyan AE, Hamilton DR, Jones JA, Melton S, Whitson PA, Kirkpatrick AW, Martin D, Dulchavsky SA. FAST at MACH 20: clinical ultrasound aboard the International Space Station. J Trauma. 2005 Jan;58(1):35-9.

[12] Chiao L, Sharipov S, Sargsyan AE, Melton S, Hamilton DR, McFarlin K, Dulchavsky SA. Ocular examination for trauma; clinical ultrasound aboard the International Space Station. J Trauma. 2005 May;58(5):885-9.

[13] Kozerenko O.P., Gushin V.I., Sled A.D. et.al. Some problems of group interaction in prolonged space flight. J. of Human Perform. In Extreme Environ. V. 4, № 1, p. 146$152,1999$.

[14] Sipes WE, Vander Ark ST. Operational behavioral health and performance resources for international space station crews and families. Aviat Space Environ Med. 2005 Jun;76(6 Suppl):B36-41. 


\section{Figure Captions}

Figure 1: ISS multilateral medical boards, panels, and working groups. MMIOCB -

Multilateral Mission Integration and Operations Control Board; MCOP - Multilateral Crew Operations Panel.

Figure 2: The ISS Multilateral medical certification flowchart.

Figure 3: The four layers of the ISS medical status and readiness review process. 


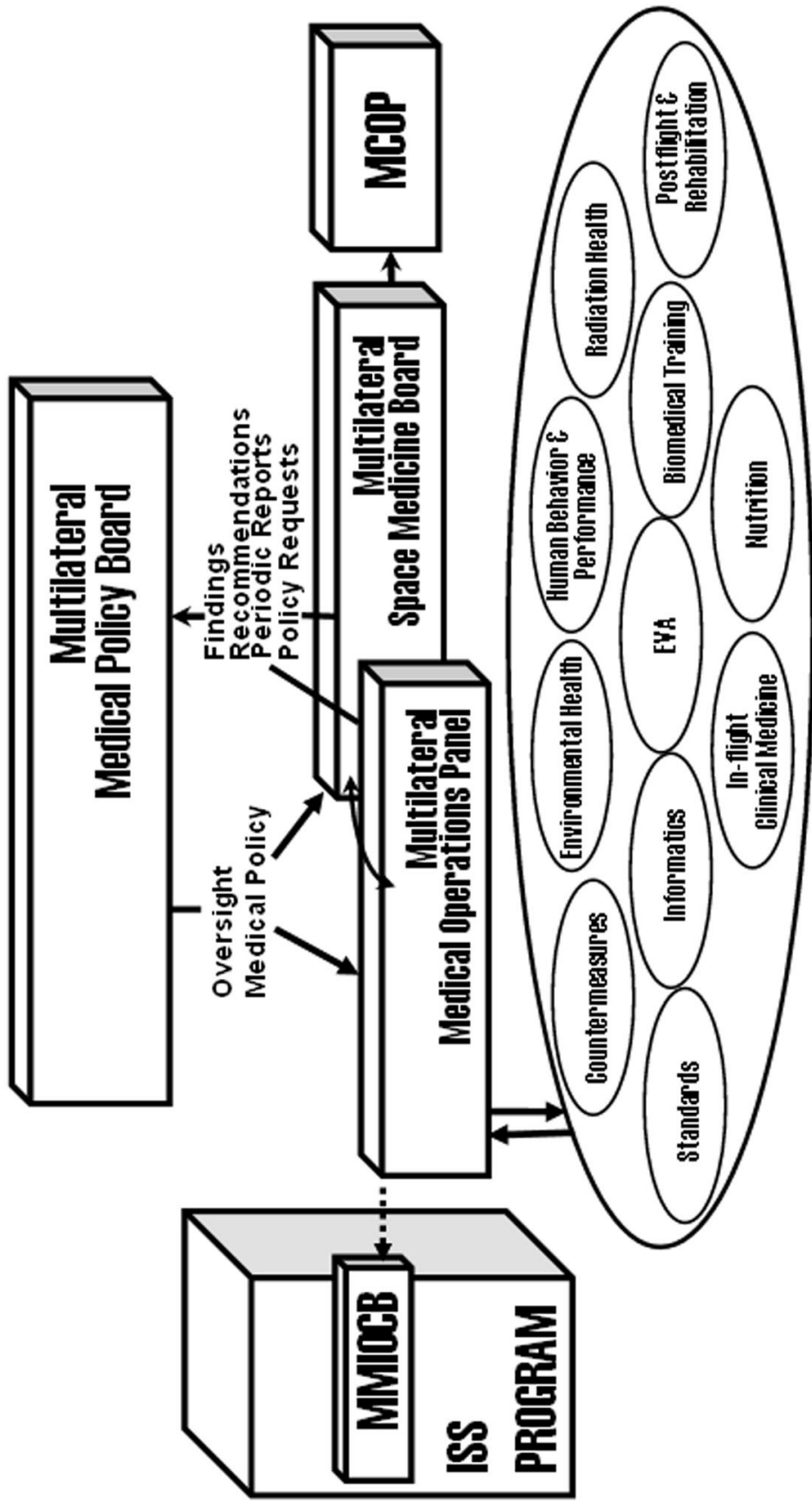




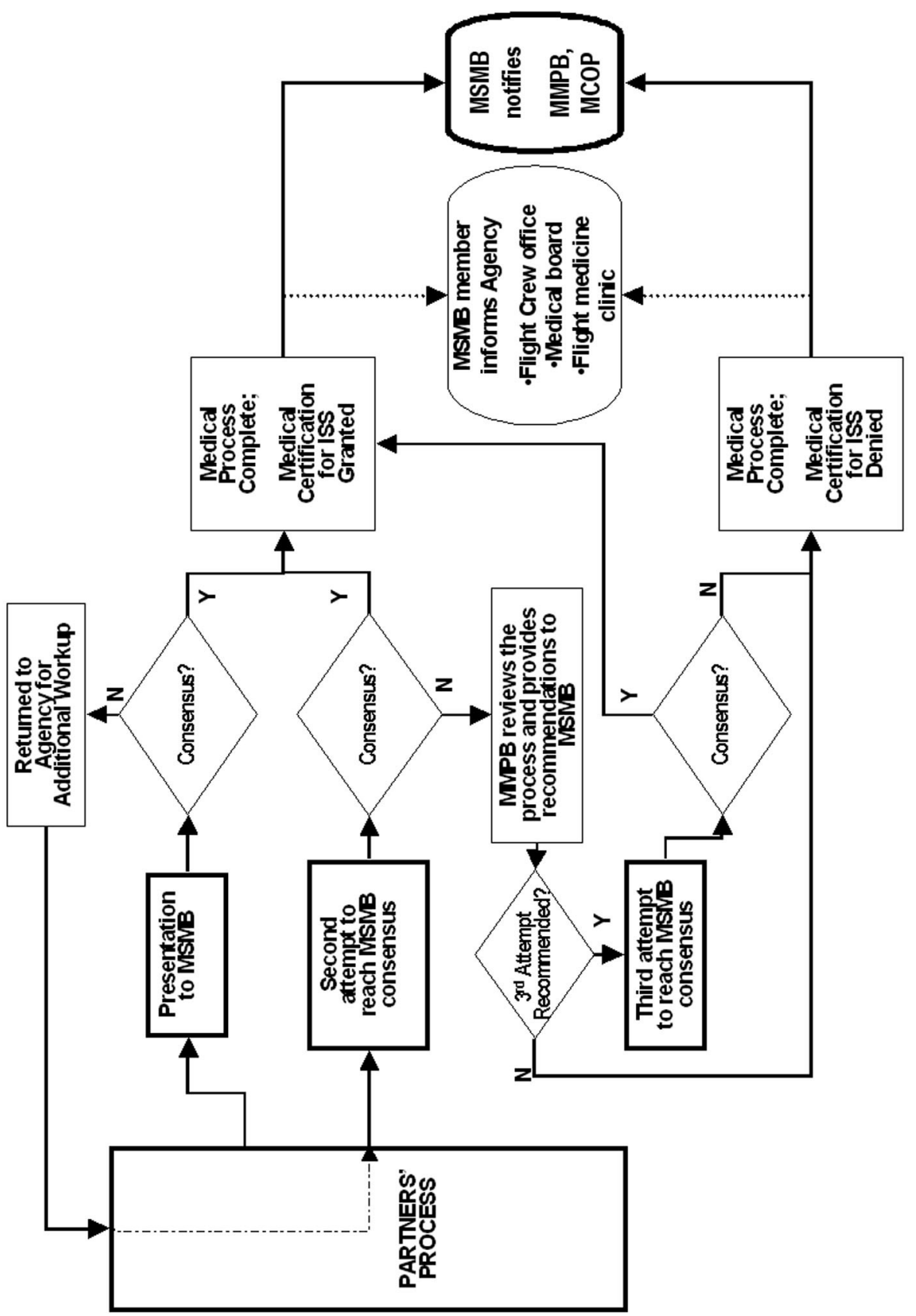




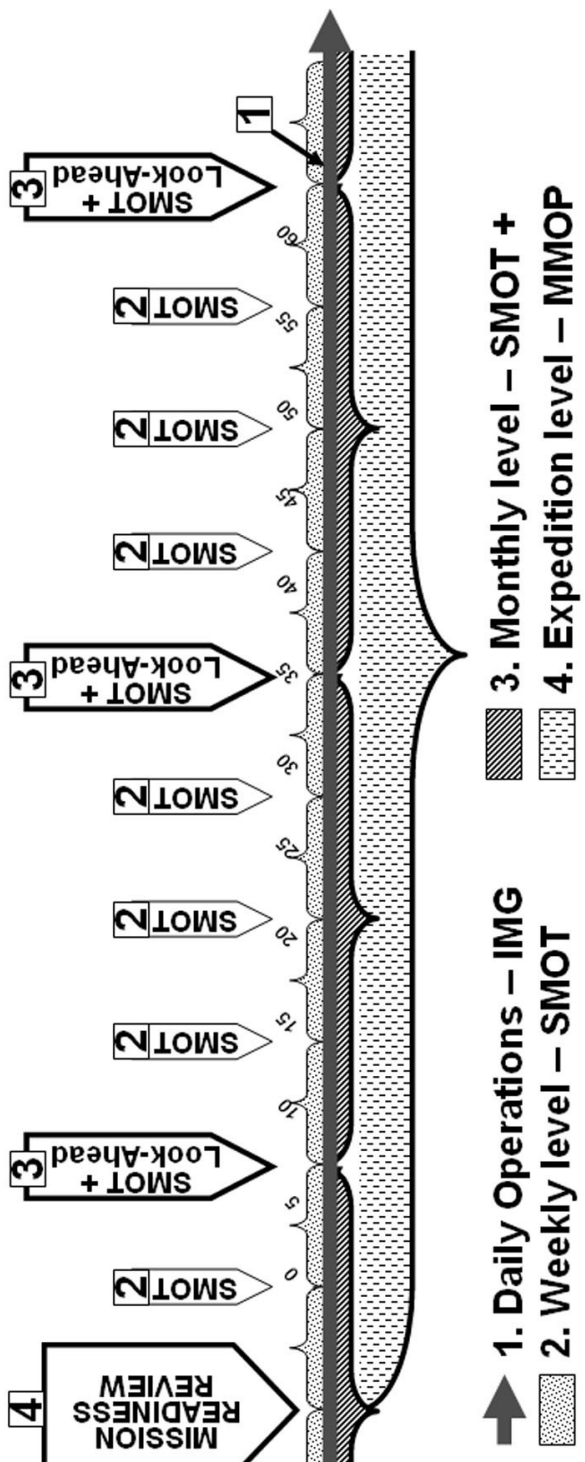


Table 1. Areas of emphasis in the MMOP mission readiness review process

\begin{tabular}{|c|c|}
\hline Aspect of Readiness & Primary Considerations \\
\hline Crew Medical Certification & $\begin{array}{l}\text { MSMB status of medical certification for all crewmembers (new ISS crew and } \\
\text { visiting short-duration crewmembers). Russian or NASA approval for special medical } \\
\text { aspects of the visiting vehicle (Soyuz or Shuttle). }\end{array}$ \\
\hline Crew Training & Completion of all required biomedical training tasks. \\
\hline $\begin{array}{l}\text { Evaluation and Monitoring of } \\
\text { Crew Health and Performance }\end{array}$ & $\begin{array}{l}\text { The availability of necessary capabilities and equipment to perform basic evaluation } \\
\text { and monitoring of health and performance of the crew on the station. }\end{array}$ \\
\hline $\begin{array}{l}\text { Diagnostic and Treatment } \\
\text { Capabilities }\end{array}$ & $\begin{array}{l}\text { The availability of equipment and capabilities to adequately diagnose and treat routine } \\
\text { medical and dental problems and to perform cardiopulmonary resuscitation (CPR) and } \\
\text { basic life support (BLS) procedures. }\end{array}$ \\
\hline The ISS Environment & $\begin{array}{l}\text { Water and air quality for permanent human presence, existence of plans and } \\
\text { equipment for long-duration monitoring of environmental parameters. }\end{array}$ \\
\hline Sustaining Human Health & $\begin{array}{l}\text { The availability of capabilities and equipment to implement and monitor operational } \\
\text { in-flight measures to maintain the human health and to mitigate undesirable effects of } \\
\text { space flight. Adequacy reserves of provisions to assure crew dietary needs. }\end{array}$ \\
\hline $\begin{array}{l}\text { Specific Areas Needing } \\
\text { Attention }\end{array}$ & $\begin{array}{l}\text { Requests to the ISS Program to recognize areas of concern or special importance as } \\
\text { high-priority areas for the mission. Example: request for "continued attention of the } \\
\text { ISS Program to maintaining the functionality of all exercise countermeasures devices } \\
\text { (RED, TVIS, VB-3 with force loaders, CEVIS, BD-1), as to a high-priority Program } \\
\text { goal for sustaining crew health and performance throughout the expedition." }\end{array}$ \\
\hline Summary & $\begin{array}{l}\text { An MMOP declaration regarding the absence or presence of medical constraints for } \\
\text { the mission, per the readiness criteria in the current version of the ISS MORD. } \\
\text { Example: "At the time of this review, no medical constraints exist to ISS } 11 \\
\text { Expedition and the } 10 S \text { mission, per the agreed upon readiness requirements of ISS } \\
\text { MORD Rev B. The MMOP finds that the crew is fully prepared for this flight and the } \\
\text { ISS is safe and operationally ready to support the arrival of the crew and the } \\
\text { performance of their mission." }\end{array}$ \\
\hline
\end{tabular}

